\title{
Epidémiologie de la tuberculose et de la brucellose des bovins en zone périurbaine d'Abéché, Tchad
}

\author{
A. Delafosse ${ }^{1 *}$ F. Goutard ${ }^{2}$ E. Thébaud ${ }^{1}$
}

\section{Mots-clés}

Bovin - Mycobacterium bovis Mycobacterium avium - Tuberculose Brucellose - Epidémiologie - Tchad.
1. Laboratoire de Farcha, BP 433, N'djaména, Tchad

2. Ecole nationale vétérinaire, 23 chemin des Capelles, 31076 Toulouse, France

* Auteur pour la correspondance

Tél./fax : +33 (0)5 45617257 ; e-mail : delaf@wanadoo.fr

\section{Résumé}

Cette étude a eu pour objectif de préciser l'épidémiologie de la tuberculose et de la brucellose des bovins dans le bassin laitier d'Abéché (préfecture du Ouaddaï, Tchad oriental). La population étudiée a été constituée d'animaux de plus d'un an pour la tuberculose et de femelles de plus d'un an pour la brucellose. L'échantillon a été sélectionné à l'aide d'un sondage à deux degrés (village puis éleveur). Le diagnostic a été réalisé par intradermotuberculination (simple puis comparative) pour la tuberculose et par une analyse sérologique [épreuve à l'antigène tamponné (EAT), puis fixation du complément (FC)] pour la brucellose. Un questionnaire destiné à caractériser l'élevage a été systématiquement rempli et un recueil de commémoratifs a été réalisé sur les animaux sélectionnés. Ces facteurs ont été inclus dans une analyse statistique multivariée. Huit cent quarante-huit animaux répartis dans 58 cheptels ont été testés par intradermotuberculination simple et 151 par intradermotuberculination comparative. Les prévalences réelles des infections à Mycobacterium bovis et à $M$. avium dans la population étudiée ont été évaluées respectivement à $0,8 \pm 0,6$ p. 100 et à $2,0 \pm 0,9$ p. $100(\alpha=5$ p. 100). La prévalence cheptel de I'infection à $M$. bovis a été évaluée à $12,4 \pm 8,5$ p. 100 et celle de l'infection à M. avium à $17,8 \pm 9,8$ p. 100 . Pour le diagnostic de la brucellose, 634 animaux répartis dans 56 cheptels ont été testés par EAT. Trente-cinq échantillons provenant de 21 cheptels ont été analysés par FC. La prévalence réelle de la brucellose a été évaluée à $2,6 \pm 1,2$ p. $100(\alpha=5$ p. 100). La prévalence cheptel a été estimée à $20,0 \pm 10,5$ p. 100 . Les principaux facteurs de risques identifiés pour la tuberculose bovine ont été un âge élevé, l'origine de l'animal (naissance en dehors du cheptel) et la garde d'animaux appartenant à d'autres éleveurs. Les principaux facteurs de risques identifiés pour la tuberculose aviaire ont été le nombre de volailles et la nature des bouviers (enfants de l'éleveur). Les principaux facteurs de risques identifiés pour la brucellose ont été l'ethnie (Arabe) et la nature des bouviers (enfants de l'éleveur). La tuberculose et la brucellose bovines semblent se développer lentement dans de nombreux cheptels, les conditions de l'élevage extensif limitant la diffusion au sein des cheptels contaminés. Ce profil épidémiologique permet une éradication de ces zoonoses à condition d'y associer un contrôle des animaux avant leur introduction dans les cheptels assainis. Cette approche pourrait être retenue en cas de développement de la production laitière en périphérie d'Abéché.

Le Tchad oriental est l'une des grandes zones d'élevage du pays (37). Le troupeau bovin de cette région est majoritairement transhumant mais un élevage sédentaire existe, en particulier à la périphérie des villes. Du fait de sa faible importance numérique cet élevage a été très peu étudié et est donc mal connu. Il constitue pourtant la principale source d'approvisionnement des villes en lait (et produits dérivés) en saison sèche. 
La tuberculose et la brucellose bovines sont des zoonoses majeures qui peuvent avoir un impact important sur la santé publique, la transmission se faisant généralement par la consommation de lait cru contaminé $(1,6,26)$. De plus, ces maladies peuvent avoir une incidence économique notable lorsqu'elles sont répandues. Ainsi, les pertes liées à la tuberculose en France, avant l'application des mesures de lutte, ont été estimées à 3 p. 100 de la production bovine (21). De même, des travaux effectués dans la région du lac Tchad ont montré que près de la moitié des femelles infectées par la brucellose avortent ce qui, en cas de forte prévalence, réduit de manière importante la fertilité du cheptel $(34,35,36)$.

$\mathrm{Au}$ Tchad oriental, la situation de ces zoonoses est mal connue. La tuberculose est fréquemment suspectée à l'abattoir d'Abéché mais l'existence de la maladie n'a jamais été confirmée par des examens de laboratoire. Une enquête sur la brucellose menée dans les années 50 a confirmé l'existence de la maladie (31). Plus récemment, une prévalence de 2,1 p. 100 à l'épreuve à l'antigène tamponné a été observée sur un échantillon de 554 dromadaires. Des antécédents d'avortements ont été signalés chez 50,0 p. 100 des femelles pubères séropositives (7).

L'étude a eu pour objectif d'évaluer l'importance de la tuberculose et de la brucellose bovines dans la zone périphérique d'Abéché, principale ville du Tchad oriental, et de préciser l'épidémiologie de ces maladies. Elle a été réalisée sur un financement du Fonds français d'aide et de coopération (Fac) dans le cadre du projet d'Appui au secteur de l'élevage au Tchad oriental (Aseto).

\section{MATERIEL ET METHODES}

\section{Cadre de l'enquête}

Les agro-éleveurs sédentaires ou semi-sédentaires installés en périphérie d'Abéché sont d'ethnies Arabe ou Maba et possèdent le plus souvent de petits cheptels (22 têtes en moyenne) (40). Les éleveurs sont installés dans des villages regroupant plusieurs familles ou dans des campements, ou ferik, lorsqu'il s'agit de nomades en voie de sédentarisation. La population bovine sédentaire a été évaluée en 1999 à 540000 têtes pour le Tchad oriental dont 163000 pour la seule sous-préfecture d'Abéché rural (9). Une zone de 30 kilomètres de rayon, correspondant approximativement au bassin laitier d'Abéché, a été retenue pour l'enquête (figure 1).

\section{Population étudiée et méthode d'échantillonnage}

La population étudiée a été constituée des troupeaux de plus de cinq têtes de bovins, situés en périphérie d'Abéché. Il a été arbitrairement décidé de limiter la population étudiée, d'une part, aux animaux de plus d'un an pour l'étude portant sur la tuberculose et, d'autre part, aux femelles de plus d'un an pour l'étude portant sur la brucellose. Les mâles ont été exclus de l'étude sur la brucellose en raison de leur faible impact sur l'épidémiologie de la maladie (1).

La population étudiée n'étant pas connue, il a été décidé d'utiliser une méthode d'échantillonnage ne nécessitant pas de recensement exhaustif des individus. Un sondage à deux degrés a été retenu : le premier degré a été constitué par le village et le second par l'éleveur. Une base de sondage regroupant tous les villages de la zone d'étude a été constituée à partir d'une carte Ign au $200000^{\mathrm{e}}$. Par ailleurs, une stratification sur la distance séparant le village de la ville d'Abéché a été réalisée afin d'améliorer la représentativité de l'échantillon. Deux strates ont ainsi été définies : dans la strate 1, les villages étaient dans un rayon de $10 \mathrm{~km}$ et, dans la strate 2 , les villages étaient dans un rayon de 10 à $30 \mathrm{~km}$. Quatre-vingt-douze villages répartis dans les deux strates ont été recensés : 12 dans la première et 80 dans la seconde. Un tirage aléatoire a été réalisé avec un

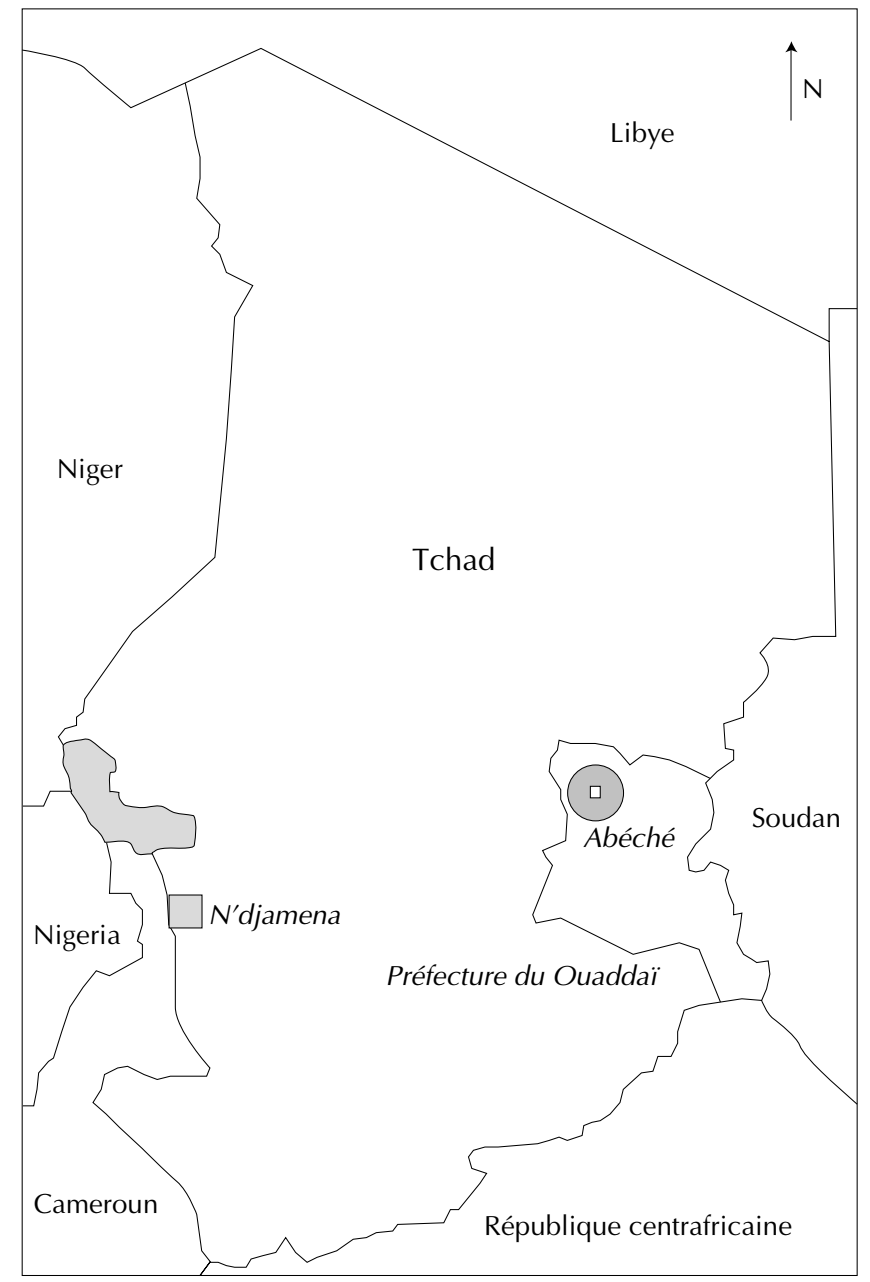

Zone enquêtée

Figure 1 : zone enquêtée.

taux de sondage identique dans chaque strate. Dans chaque village sélectionné, un ou deux éleveurs (en fonction de l'importance du site) ont été choisis au hasard. Tous les animaux des cheptels sélectionnés qui répondaient aux critères d'inclusion ont été retenus.

\section{Recueil et traitement des données}

Un questionnaire destiné à caractériser l'élevage a été systématiquement rempli. Un recueil de commémoratifs a été réalisé sur tous les animaux qui ont fait l'objet d'un diagnostic de tuberculose ou de brucellose. L'âge, l'origine, l'état d'entretien (estimé par l'enquêteur par une note allant de 1 pour « très mauvais » à 5 pour « très bon »), les antécédents d'avortement ou de pathologie respiratoire ont été ainsi relevés.

\section{Méthodes de diagnostic}

\section{Tuberculose}

La méthode utilisée a été l'injection par voie intradermique de tuberculine. Le diagnostic a été réalisé dans un premier temps par intradermotuberculination simple (Ids). C'est une méthode dont la sensibilité $(\mathrm{Se})$ et la spécificité $(\mathrm{Sp})$ sont supérieures à 90 p. 100 $(1,21)$. Elle consiste à injecter, dans l'épaisseur du derme de l'encolure, de la tuberculine $(0,1 \mathrm{ml}$ de tuberculine Ppd titrée à 20000 Uct/ml) et à apprécier, au bout de $72 \mathrm{~h}$, la réaction au point d'injection. L'augmentation du pli de peau a été évaluée à l'aide d'un cutimètre à ressort $\left(\right.$ Hauptner $\left.^{\circledR}\right)$. Le résultat a été considéré 
comme positif lorsque l'épaississement du pli de peau a été supérieur ou égal à $4 \mathrm{~mm}$, comme douteux lorsqu'il a été supérieur ou égal à $2 \mathrm{~mm}$ et inférieur à $4 \mathrm{~mm}$, et négatif lorsqu'il a été inférieur à $2 \mathrm{~mm}$. Les animaux positifs ou douteux ont été identifiés à l'aide de boucles auriculaires.

Dans un deuxième temps, le diagnostic a été réalisé par intradermotuberculination comparative (Idc). C'est une méthode dont la spécificité est excellente (>95 p. 100) mais qui est peu sensible (50 à 80 p. 100) (21, 33). Elle consiste à injecter dans l'épaisseur du derme, au niveau de l'encolure, des tuberculines bovines $(0,1 \mathrm{ml}$ de tuberculine purifiée Ppd titrée à $20000 \mathrm{Uct} / \mathrm{ml}$ ) et aviaire (titre $25000 \mathrm{UI} / \mathrm{ml}$ ) en deux points séparés de $20 \mathrm{~cm}$ et à apprécier, au bout de $72 \mathrm{~h}$, les réactions aux points d'injections. L'Ids provoquant une baisse de la réactivité des animaux sensibilisés, un délai minimum de 40 jours entre les deux tests a été respecté. L'Idc a été réalisée sur tous les animaux bouclés (positifs et douteux à l'Ids) qui ont pu être retrouvés. L'utilisation de cette technique a visé à différencier les infections par M. bovis des infections par d'autres mycobactéries (notamment celles du groupe aviaire). L'interprétation du résultat a été réalisée par la différence entre les épaississements des plis de peau après injection des tuberculines bovine (B) et aviaire (A). Un épaississement $(B-A) \geq 4 \mathrm{~mm}$ impliquait une réaction positive à la tuberculine bovine ; un épaississement $(B-A) \geq 2 \mathrm{~mm}$ et $<4 \mathrm{~mm}$ impliquait une réaction douteuse à la tuberculine bovine ; un épaississement $(\mathrm{A}-\mathrm{B}) \geq 4 \mathrm{~mm}$ impliquait une réaction positive à la tuberculine aviaire ; un épaississement $(\mathrm{A}-\mathrm{B}) \geq 2 \mathrm{~mm}$ et $<4 \mathrm{~mm}$ impliquait une réaction douteuse à la tuberculine aviaire ; un épaississement $(B-A)>-2 \mathrm{~mm}$ et $<2 \mathrm{~mm}$ impliquait une réaction atypique.

\section{Brucellose}

Les prises de sang ont été effectuées à la veine jugulaire sur tube sec, chaque tube ayant été identifié avec le numéro de l'éleveur et le numéro de l'animal. Les sérums ont été prélevés à l'aide de pipettes jetables stériles après coagulation du caillot.

Le diagnostic a été réalisé avec une épreuve à l'antigène tamponné (EAT) ou test au rose bengale. C'est un test rapide, simple, économique, réputé très sensible ( $\geq 95 \mathrm{p}$. 100) et relativement peu spécifique ( $\geq 90$ p. 100) (1). Afin de déceler les faux positifs, une réaction de fixation du complément a été effectuée sur les prélèvements positifs ou douteux au rose bengale. La réaction a été considérée comme positive lorsque le titre de sérum a été supérieur à 20 Ucees $/ \mathrm{ml}$. Ce test est considéré comme très sensible ( $\geq 95$ p. 100) et très spécifique ( $\geq 95$ p. 100) (1).

\section{Analyse statistique}

L'analyse statistique a été réalisée pour prendre en compte simultanément divers facteurs pouvant influer sur la prévalence de la tuberculose et de la brucellose. Les variables d'intérêts, codées en présence/absence, ont été la positivité aux tests de diagnostic (tuberculose : (1) Idc positif à la tuberculine bovine, (2) Idc positif à la tuberculine aviaire, (3) Idc réaction atypique ; brucellose : positif en fixation du complément). Les variables explicatives ont été des caractéristiques individuelles et collectives (tableau I).

La sélection des effets fixes a été réalisée par une régression logistique ordinaire à partir de pré-modèles comprenant toutes les variables explicatives et toutes les interactions du premier ordre. Le critère de sélection a été une procédure descendante visant à minimiser le critère d'Akaike (Aic, Akaike Information Criterion) et à simplifier les modèles afin d'en faciliter l'interprétation. L'ajustement a été réalisé avec le logiciel $\mathrm{R}^{\circledR}$ (modules Glm et Gee).

Les effets fixes ont ensuite été inclus dans des modèles Gee (équations généralisées d'estimation) permettant de prendre en compte la non-indépendance des unités statistiques regroupées au sein de troupeaux $(23,32)$.

\section{Tableau}

Variables explicatives inclues dans I'analyse statistique

\begin{tabular}{|c|c|c|c|}
\hline Nature & Intitulé & Type & Modalités \\
\hline $\begin{array}{l}\text { Caractéristiques individuelles } \\
\text { (animaux) }\end{array}$ & $\begin{array}{l}\text { Origine } \\
\text { Age } \\
\text { Sexe } \\
\text { Etat d'entretien }\end{array}$ & $\begin{array}{l}\text { Qualitative nominale } \\
\text { Quantitative } \\
\text { Qualitative nominale } \\
\text { Qualitative ordinale }\end{array}$ & $\begin{array}{l}\text { Né dans le troupeau/autres } 1 \\
- \\
\text { Mâle/femelle } \\
\text { Note d'état (1 à } 5)\end{array}$ \\
\hline $\begin{array}{l}\text { Localisation } \\
\text { (par rapport à Abéché) }\end{array}$ & $\begin{array}{l}\text { Latitude } \\
\text { Longitude } \\
\text { Eloignement }\end{array}$ & $\begin{array}{l}\text { Qualitative nominale } \\
\text { Qualitative nominale } \\
\text { Qualitative nominale }\end{array}$ & $\begin{array}{l}\text { Nord/sud } \\
\text { Est/ouest } \\
>10 \mathrm{~km} / \leq 10 \mathrm{~km}\end{array}$ \\
\hline $\begin{array}{l}\text { Caractéristiques du cheptel } \\
\text { (effectifs) }\end{array}$ & $\begin{array}{l}\text { Bovins } \\
\text { Petits ruminants } \\
\text { Equidés (ânes, chevaux) } \\
\text { Volailles }\end{array}$ & $\begin{array}{l}\text { Quantitative } \\
\text { Quantitative } \\
\text { Quantitative } \\
\text { Quantitative }\end{array}$ & $\begin{array}{l}- \\
- \\
- \\
-\end{array}$ \\
\hline Caractéristiques éleveur/pratiques & $\begin{array}{l}\text { Ethnie } \\
\text { Habitat } \\
\text { Confiage }^{2} \\
\text { Déplacements saisonniers } \\
\\
\text { Logement nuit } \\
\text { Surveillance cheptel }\end{array}$ & $\begin{array}{l}\text { Qualitative nominale } \\
\text { Qualitative nominale } \\
\text { Qualitative nominale } \\
\text { Qualitative nominale } \\
\text { Qualitative nominale } \\
\text { Qualitative nominale }\end{array}$ & $\begin{array}{l}\text { Maba/Arabe } \\
\text { Campement/village } \\
\text { Oui/non } \\
\text { Oui/non } \\
\text { Parc/piquet/liberté } \\
\text { Eleveur/autres }{ }^{4}\end{array}$ \\
\hline
\end{tabular}




\section{RESULTATS}

\section{Epidémiologie descriptive}

Les résultats ont été exprimés pour chaque maladie aux deux niveaux étudiés : animal et cheptel. La prévalence cheptel a été définie comme le rapport du nombre de cheptels infectés (comportant au moins un cas) sur le nombre total de cheptels.

\section{Tuberculose}

\section{- Résultats bruts}

Au niveau individuel, huit cent quarante-huit animaux, provenant de 58 cheptels, ont été testés par Ids. Trente et un bovins (3,7 p. 100) ont présenté une réaction positive et 165 (19,5 p. 100) une réaction douteuse. Cent cinquante et un bovins (dont 23 positifs et 128 douteux à l'Ids) ont pu être retrouvés puis testés par Idc. Huit (5,3 p. 100) ont présenté une réaction positive et $9(6,0 \mathrm{p}$. 100) une réaction douteuse à la tuberculine bovine. Onze $(7,3$ p. 100) ont présenté une réaction positive et $18(11,9$ p. 100$)$ une réaction douteuse à la tuberculine aviaire. Cent cinq (69,5 p. 100) ont présenté une réaction atypique. Sur 23 animaux positifs à l'Ids, 4 (17,4 p. 100) ont été positifs à la tuberculine bovine et $3(13,0$ p. 100) à la tuberculine aviaire. Sur 128 animaux douteux à l'Ids, 4 (3,1 p. 100) ont été positifs à la tuberculine bovine et $8(6,2$ p. 100) à la tuberculine aviaire. Aucun animal positif ou douteux à la tuberculine bovine n'a eu des symptômes pouvant être associés à la tuberculose (toux quinteuse, cachexie).

Au niveau groupe, le nombre moyen d'Ids réalisées par cheptel a été de 14,6 (4 au minimum et 30 au maximum ; écart-type $=7$ ). Des réactions douteuses ont été relevées dans 50 cheptels $(86,2$ p. 100) et des réactions positives dans 21 (36,2 p. 100). Cinquante et un cheptels $(87,9$ p. 100) ont présenté au moins un animal positif ou douteux à l'Ids. Quarante-huit cheptels, dont 20 avaient présenté au moins une réaction positive et 47 au moins une réaction douteuse à l'Ids, ont pu être testés par Idc. Le nombre moyen d'Idc par cheptel a été de 3,1 (1 au minimum et 11 au maximum, écart-type =2). Sept cheptels (14,6 p. 100) ont présenté au moins une réaction positive et 8 (16,7 p. 100) au moins une réaction douteuse à la tuberculine bovine. Neuf cheptels (18,7 p. 100) ont présenté au moins une réaction positive et $13(27,1 \mathrm{p} .100)$ au moins une réaction douteuse à la tuberculine aviaire. Des réactions atypiques ont été observées dans 39 cheptels (81,2 p. 100). Sur 20 cheptels positifs à l'Ids, $6(30,0 \mathrm{p}$. 100) ont été positifs à la tuberculine bovine et $3(15,0$ p. 100) à la tuberculine aviaire. Sur 47 cheptels douteux à l'Ids, 6 (12,5 p. 100) ont été positifs à la tuberculine bovine et $9(19,1$ p. 100) à la tuberculine aviaire.

- Estimation de la prévalence des infections

à Mycobacterium bovis et à M. avium dans la population étudiée

Les valeurs théoriques des prévalences apparentes mesurées avec l'Ids et l'Idc peuvent être obtenues avec les formules (38) :

$\mathrm{P}_{\text {Ids }}=\mathrm{P}_{\text {réelle }} \mathrm{x}\left(\mathrm{Se}_{\mathrm{Ids}}+\mathrm{Sp}_{\mathrm{Ids}}-1\right)+\left(1-\mathrm{Sp}_{\mathrm{Ids}}\right)$

$\mathrm{P}_{\mathrm{Idc}}=\mathrm{VPP}_{\mathrm{Ids}} \mathrm{x}\left(\mathrm{Se}_{\mathrm{Idc}}+\mathrm{Sp}_{\mathrm{Idc}}-1\right)+\left(1-\mathrm{Sp}_{\mathrm{Idc}}\right)$

$\operatorname{VPP}_{\text {Ids }}=\left(\mathrm{Se}_{\text {Ids }} \times \mathrm{P}_{\text {réelle }}\right) /\left[\left(\mathrm{Se}_{\text {Ids }} \times \mathrm{P}_{\text {réelle }}\right)+\left(1-\mathrm{Sp}_{\text {Ids }}\right)\left(1-\mathrm{P}_{\text {réelle }}\right)\right]$

où $\mathrm{P}_{\mathrm{Ids}}=$ prévalence apparente mesurée par Ids dans l'échantillon initial (M. bovis : réactions positives Ids ; $M$. avium : réactions douteuses Ids) $; \mathrm{P}_{\mathrm{Idc}}=$ prévalence apparente mesurée par Idc (M. bovis : dans groupe positif Ids ; M. avium : dans groupe douteux Ids) $; \mathrm{P}_{\text {réelle }}=$ prévalence réelle dans l'échantillon initial ; $\mathrm{Se}_{\mathrm{Ids}}=$ sensibilité Ids $; \mathrm{Sp}_{\mathrm{Ids}}=$ spécificité Ids $; \mathrm{Se}_{\mathrm{Idc}}=$ sensibilité Idc $; \mathrm{Sp}_{\text {Idc }}=$ spécificité Idc $; \mathrm{VPP}_{\text {Ids }}=$ valeur prédictive positive de l'Ids.

Dès lors, les prévalences réelles des infections à $M$. bovis et M. avium et les couples sensibilité/spécificité des tests ont pu être évalués en déterminant, par itérations successives, les valeurs qui minimisent l'écart entre les prévalences apparentes théoriques et observées lors de l'enquête (tableau II).

\section{Tableau II}

Prévalences réelles des infections à Mycobacterium bovis et M. avium dans la population étudiée (estimations obtenues à partir d'hypothèses sur les valeurs intrinsèques des tests)

Prév. réelle estimée (\%)

Intervalle confiance $(\alpha=5 \%)$

\section{Intradermoréaction simple (Ids)}

Sensibilité

Spécificité

Prév. apparente $(\%)^{1}$

\section{Intradermoréaction comparative (Idc)}

Sensibilité

Spécificité

Prév. apparente $(\%)^{2}$

\begin{tabular}{cc}
\multicolumn{2}{c}{ M. bovis } \\
\hline Individu & Troupeau \\
\hline 0,8 & 12,4 \\
{$[0,2-1,4]$} & {$[3,9-20,9]$} \\
& \\
& \\
0,90 à 1,00 & $\geq 0,94$ \\
0,94 & 0,94 \\
0,90 à 1,00 & $\leq 0,97$ \\
0,97 & 0,72 \\
3,66 & 36,20 \\
3,73 & 36,18
\end{tabular}

$\begin{array}{lcc}\text { Hypothèse } & 0,90 \text { à } 1,00 & \geq 0,94 \\ \text { Estimée } & 0,94 & 0,94 \\ \text { Hypothèse } & 0,90 \text { à } 1,00 & \leq 0,97 \\ \text { Estimée } & 0,97 & 0,72 \\ \text { Observée } & 3,66 & 36,20 \\ \text { Estimée } & 3,73 & 36,18\end{array}$

0,90 à 1,00
0,91
0,80 à 1,00
0,82
19,46
19,46

$\geq 0,91$

0,92

$\leq 0,83$

0,15

86,21

86,25

$\begin{array}{lcccc}\text { Hypothèse } & 0,50 \text { à } 0,80 & \geq 0,82 & 0,50 \text { à } 0,80 & \geq 0,475 \\ \text { Estimée } & 0,82 & 0,82 & 0,475 & 0,62 \\ \text { Hypothèse } & 0,95 \text { à } 1,00 & \leq 0,99 & 0,95 \text { à } 1,00 & \leq 0,98 \\ \text { Estimée } & 0,99 & 0,94 & 0,98 & 0,91 \\ \text { Observée } & 17,39 & 30,00 & 6,25 & 19,15 \\ \text { Estimée } & 17,34 & 30,48 & 6,26 & 19,06\end{array}$

${ }^{1}$ Mycobacterium bovis : réactions positives à l'Ids ; $M$. avium : réactions douteuses à l'Ids

${ }^{2}$ Obtenue à partir des groupes positif à l'Ids (M. bovis) et douteux à l'Ids (M. avium) 
Il a été nécessaire, pour ce calcul, d'émettre des hypothèses sur la valeur du couple sensibilité/spécificité des tests aux deux niveaux étudiés (individu et cheptel). La sensibilité cheptel a été considérée comme supérieure ou égale à la sensibilité individuelle et la spécificité cheptel comme inférieure ou égale à la spécificité individuelle (41).

\section{Brucellose}

- Résultats bruts

Au niveau individuel (animal), six cent trente-quatre prises de sang ont été effectuées dans 56 cheptels. Vingt-quatre (4,0 p. 100) et 13 (2,0 p. 100) bovins ont été trouvés, respectivement, positifs et douteux à l'EAT. Sur ces 37 échantillons, 35 ( 23 positifs et 12 douteux) ont été testés par la fixation du complément (2 sérums hémolysés n'ont pu être analysés). Vingt bovins, dont 16 ont été positifs à l'EAT et 4 douteux, ont été trouvés positifs au test de fixation du complément.

Des antécédents d'avortements ont été signalés chez 35,0 p. 100 (7 sur 20) des vaches (> 4 ans) positives au test de fixation du complément, alors que cette proportion n'a été que de 7,0 p. 100 (42 sur 597) chez celles qui ont été négatives à l'EAT (différence significative, test du $\left.\chi^{2}, \mathrm{p} \leq 0,001\right)$. Aucune association n'a été relevée entre la positivité à la fixation du complément et la présence d'hygromas.

Au niveau du groupe (cheptel), le nombre moyen d'examens sérologiques par cheptel a été de 11,3 (écart-type $=5,0 ;$ minimum $=3$; maximum $=27)$. Vingt et un cheptels sur 56 (37,5 p. 100) ont présenté au moins une réaction positive et/ou douteuse à l'EAT et 14 (25,0 p. 100) au moins une réaction positive. Onze cheptels sur $21(52,4$ p. 100) ont présenté au moins un cas de réaction positive à la FC. Ils sont tous provenus de cheptels qui avaient eu au moins une réaction positive à l'EAT. Le nombre moyen de FC par cheptel a été de 1,7 (écart-type $=1,0 ;$ minimum $=1 ;$ maximum $=5$ ).

- Estimation de la prévalence de la brucellose dans la population étudiée

La prévalence réelle de la brucellose a été estimée en utilisant la même procédure que celle décrite pour la tuberculose (tableau III).

\section{Tableau III}

Prévalence réelle de la brucellose dans la population étudiée

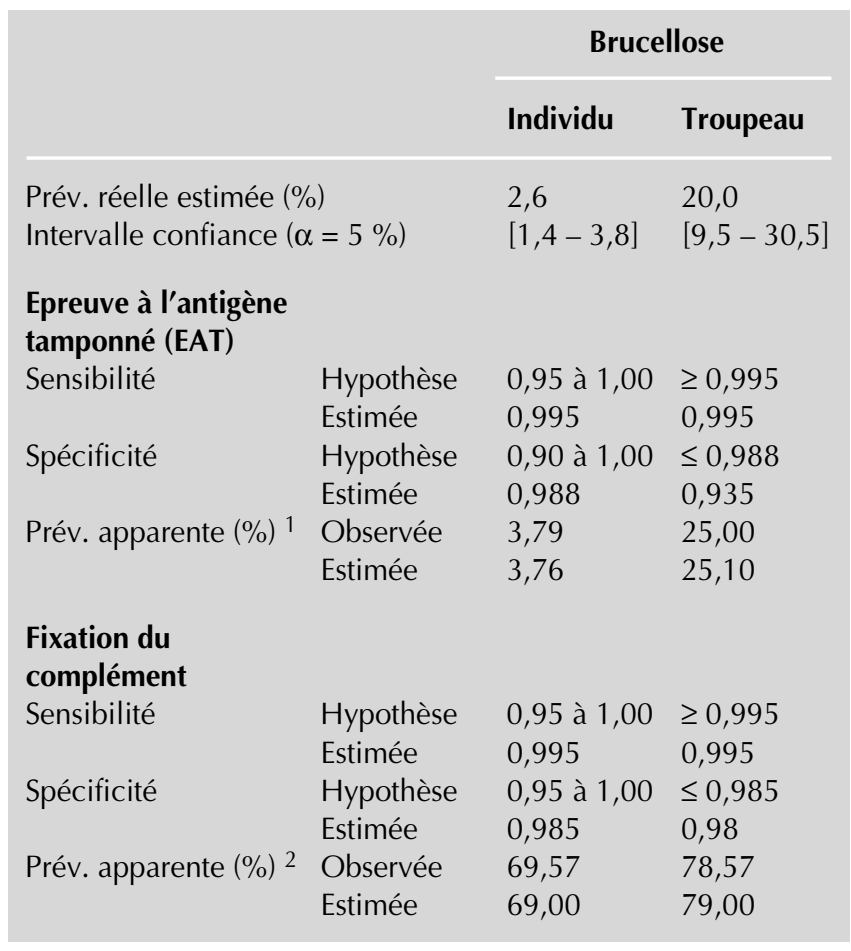

${ }^{1}$ Obtenue à partir de l'échantillon initial

${ }^{2}$ Obtenue à partir du groupe positif à l'EAT

\section{Facteurs de risques}

Le risque de positivité à la tuberculine bovine a augmenté avec l'âge et lorsque l'animal n'était pas né dans le cheptel. Il a également augmenté chez les éleveurs pratiquant le confiage (tableau IV). Le risque de positivité à la tuberculine aviaire a augmenté

\section{Tableau IV}

Facteurs de risques identifiés

\begin{tabular}{|c|c|c|c|c|c|}
\hline \multirow{2}{*}{\multicolumn{2}{|c|}{ Facteurs de risques }} & \multicolumn{4}{|c|}{ Coefficients modèles Gee } \\
\hline & & Mycobacterium bovis & M. avium & Réactions atypiques & Brucellose \\
\hline \multicolumn{6}{|l|}{ Individuels } \\
\hline Age & & $0,3278^{* *}$ & NS & $-0,4876^{* * *}$ & $-0,6415^{*}$ \\
\hline Etat & & NS & NS & $0,3667^{*}$ & NS \\
\hline Origine $(1=$ & & $-10,8867^{*}$ & NS & $-1,3611^{*}$ & NS \\
\hline \multicolumn{6}{|l|}{ Collectifs } \\
\hline \multicolumn{2}{|c|}{ Ethnie (1 = Maba) } & NS & NS & NS & $-7,4556^{* *}$ \\
\hline \multicolumn{2}{|c|}{ Habitat (1 = campement) } & NS & NS & NS & $-7,4531^{*}$ \\
\hline \multicolumn{2}{|c|}{ Surveillance cheptel ( 1 = autres) } & NS & $1,5683^{*}$ & $1,6739 * *$ & $4,4015^{* *}$ \\
\hline \multicolumn{2}{|c|}{ Confiage } & $4,5428^{* *}$ & $-1,5038^{*}$ & NS & NS \\
\hline \multirow[t]{2}{*}{ Cheptel } & Bovins & NS & NS & NS & $-0,4180^{*}$ \\
\hline & Volailles & NS & $0,0891^{*}$ & NS & NS \\
\hline Localisation & Nord & NS & NS & NS & $-7,6015^{*}$ \\
\hline
\end{tabular}


avec le nombre de volailles et lorsque l'éleveur n'assurait pas la surveillance du cheptel et ne pratiquait pas le confiage. Le risque de réactions atypiques a diminué avec l'âge et a augmenté avec la note d'état de l'animal. Il a également augmenté lorsque l'animal n'était pas né dans le cheptel et quand l'éleveur n'assurait pas la surveillance du troupeau. Le risque de positivité à la brucellose a diminué avec l'âge et a augmenté lorsque l'éleveur n'assurait pas la surveillance des animaux. L'origine ethnique de l'éleveur (Arabe), un habitat villageois, un faible nombre de bovins et une localisation méridionale par rapport à Abéché ont également augmenté ce risque.

\section{Méthodologie}

La base de sondage a été élaborée à l'aide de cartes datant des années 50 . Le paysage démographique de la région s'étant largement modifié, de nombreuses installations humaines n'ont pu être prises en compte. De plus, il a été décidé d'inclure, dans un souci de représentativité, les campements des éleveurs en cours de sédentarisation non indiqués sur les cartes. Dans quelques cas, la sélection du village a donc relevé d'un choix raisonné plutôt que d'un véritable tirage aléatoire.

Un biais de recrutement des éleveurs lié à quelques refus de participation a pu également intervenir. La lourdeur des investigations a poussé certains éleveurs à repousser d'emblée le protocole alors que d'autres, peu nombreux, se sont désistés lors de l'Idc. Par ailleurs, quelques éleveurs ont refusé les prélèvements de sang pour le diagnostic de la brucellose.

Lors de l'Ids, le pli de peau contrôle a été mesuré le jour de la lecture sur l'autre face de l'encolure. Cette pratique a pu biaiser certaines interprétations de l'Ids. La méthode d'interprétation de l'Idc est légèrement différente de celle, habituellement préconisée, qui considère la réaction comme douteuse à la tuberculine bovine lorsque la différence d'augmentation des plis de peau est comprise entre 1 et $4 \mathrm{~mm}$ (21). Le type d'interprétation utilisé ici a conduit à limiter les réactions douteuses au profit de réactions considérées comme atypiques.

Enfin, les résultats obtenus n'ont été représentatifs que des populations étudiées (zone périurbaine d'Abéché ; bovins de plus d'un an pour la tuberculose ; génisses et vaches de plus d'un an pour la brucellose) et, de ce fait, toute extrapolation à l'ensemble de la population bovine du Tchad oriental doit être évitée.

\section{Epidémiologie descriptive}

\section{Tuberculose}

L'étude a permis d'estimer à 0,8 p. 100 la prévalence réelle de l'infection des bovins par Mycobacterium bovis. Cette valeur est proche de celles relevées entre 1976 et 1991 dans divers pays sahéliens avec des prévalences apparentes à l'Idc de 1,2 p. 100 au sud de la Mauritanie (43), de 2 p. 100 au Niger (2) et de 3,9 p. 100 au nord du Burkina Faso $(38,44)$.

L'association de conditions climatiques défavorables à la survie du germe et des pratiques de l'élevage extensif, caractérisé par de faibles densités animales, pourrait expliquer la faible propagation de l'infection.

Lorsque le climat devient plus humide ou que les conditions d'élevage s'intensifient, on observe souvent une augmentation de la prévalence de la tuberculose bovine. A Madagascar et en Tanzanie, de fortes prévalences ont été relevées dans les régions où le climat et les pratiques d'élevage sont modifiés par l'altitude (19, 33). En Erythrée, pays au climat désertique, une prévalence apparente à l'Idc de 14,5 p. 100 a été observée dans les élevages laitiers périurbains (30). Au Ghana, la prévalence réelle de l'infection dans les élevages laitiers périurbains a été estimée à 13,8 p. 100 (3).

La prévalence cheptel a été estimée à 12,4 p. 100. Un décalage entre les prévalences mesurées sur les animaux et les cheptels est classiquement observé dans les conditions de l'élevage extensif. Dans le nord de la Tanzanie, une enquête portant sur deux populations distinctes, élevages traditionnels et laitiers périurbains, a permis de relever des prévalences apparentes à l'Idc de 0,6 et 0,9 p. 100 sur les animaux, alors que 21 p. 100 des troupeaux traditionnels étaient positifs contre 10 p. 100 des troupeaux laitiers (45). En Erythrée, seuls 41,7 p. 100 des cheptels laitiers étaient positifs à l'Idc alors que la prévalence individuelle était de 14,5 p. 100 (30).

Il est probable que les conditions de l'élevage extensif n'autorisent qu'une lente propagation de l'infection dans les cheptels tout en permettant le passage d'un cheptel à l'autre lors de contact au pâturage où à l'occasion de l'introduction d'animaux. A l'opposé, en élevage laitier, la forte densité animale dans les bâtiments permettrait une diffusion plus rapide au sein des cheptels contaminés alors que le cloisonnement des élevages limiterait la contagion entre cheptels.

Aucune répercussion clinique de la maladie n'a pu être enregistrée : les animaux positifs n'ont pas présenté de toux et ont généralement été en bon état d'entretien. La tuberculose a donc semblé essentiellement exister sous forme d'infection. Il est probable que les conditions difficiles d'élevage entraînent une disparition rapide des animaux malades. Cette mortalité précoce pourrait limiter les possibilités de contamination et expliquerait la faible proportion d'animaux infectés.

La prévalence réelle de la tuberculose aviaire a été estimée à 2,0 p. 100. Cette valeur a été plus faible que celles relevées au sud de la Mauritanie (avec une prévalence apparente à l'Idc de 17,2 p. 100) (43), au nord du Burkina Faso $(13,8$ p. 100) $(38,44)$ et de la Côte d'Ivoire (4,7 p. 100) (11). Cette différence est principalement liée à la méthodologie employée lors de ces travaux où toute réaction positive à la tuberculine aviaire avait été attribuée à M. avium. Aucune répercussion clinique n'a pu être enregistrée, ce qui rejoint la faible pathogénicité de M. avium pour les bovins (21).

Les animaux qui ont présenté des réactions atypiques ont pu être sensibilisés par des mycobactéries non tuberculeuses. Ce groupe comprend en premier lieu des mycobactéries du complexe terrae dont $M$. farcinogenes, agent du farcin du bœuf, et $M$. avium subsp. paratuberculosis, agent de l'entérite paratuberculeuse des bovidés $(21,22)$. A proximité de la zone de l'étude, à l'ouest du Soudan, la prévalence du farcin a été évaluée à 31,9 p. 100 (15). D'autres espèces, dont $M$. acquae, agent de la thélite nodulaire, sont également susceptibles de provoquer des infections bénignes de la peau, des mamelles, du poumon ou du foie (22).

\section{Brucellose}

La prévalence réelle de la brucellose bovine a été évaluée à 2,6 p. 100. Cette valeur a été plus faible que celles observées au Tchad entre 1976 et 1978 (34, 35, 36). Au Nord, dans la région du lac Tchad, une valeur de 25,0 p. 100 avait été relevée, par fixation du complément, sur un échantillon de 4931 femelles provenant de cheptels transhumants. Au Sud, des prévalences apparentes à l'EAT de 15,4 et 36,0 p. 100 avaient été mesurées lors d'enquêtes portant sur des cheptels sédentaires et transhumants. Cette différence tient principalement à l'objectif de ces études qui visait à explorer des foyers de brucellose abortive et non à décrire la situation épidémiologique à partir d'un échantillon retenu par tirage aléatoire. 
La prévalence obtenue ici est par ailleurs comparable à celles mesurées lors de diverses enquêtes sérologiques effectuées en Afrique du Sud et en zone sahélienne du Burkina Faso, du Nigeria et du Niger $(2,4,12,13)$.

Comme pour la tuberculose, l'intensification des méthodes d'élevage et le climat semblent avoir une influence sur l'épidémiologie de la maladie (10). En Erythrée, la prévalence apparente au rose bengale était de 8,2 p. 100 dans les cheptels laitiers périurbains contre 5,0 p. 100 dans les cheptels traditionnels (28). Au Burkina Faso et en Côte d'Ivoire, les prévalences apparentes étaient plus élevées dans les zones méridionales au climat plus humide (13). Enfin, de fortes prévalences ont été relevées dans les zones humides du Ghana, du Nigeria, du Sud Soudan et de la Somalie méridionale $(16,27,42,46)$.

La prévalence cheptel a été estimée à 20,0 p. 100. Comme pour la tuberculose, les conditions de l'élevage extensif expliquent certainement le décalage entre la prévalence individuelle et la prévalence cheptel par ailleurs fréquemment constaté dans les élevages traditionnels en Afrique tropicale (10). Ainsi, en Erythrée, le couple prévalence individuelle/prévalence cheptel a été évalué à 5,0 p. $100 / 46,1$ p. 100 dans les élevages traditionnels et à 8,2 p. 100/35,9 p. 100 dans les élevages laitiers périurbains (28).

Un tiers des vaches séropositives ont eu des antécédents d'avortements, ce qui correspond à la faible fréquence de la forme abortive de la brucellose en Afrique tropicale (10). Aucune d'elles n'a présenté d'hygroma alors qu'il s'agit d'un symptôme très fréquemment décrit dans cette zone géographique (10). Ainsi, des symptômes articulaires avaient été observés chez deux bovins séropositifs sur trois lors d'une étude menée dans la région du lac Tchad $(34,35,36)$. Cette différence pourrait s'expliquer par un défaut de détection ou par la virulence de la souche, différents biotypes de $B$. abortus ayant été isolés en Afrique (1).

\section{Facteurs de risques}

\section{Tuberculose}

Les animaux positifs à la tuberculine bovine ont généralement été âgés, ce qui rejoint les résultats obtenus dans d'autres élevages traditionnels d'Afrique tropicale $(11,19,38,43,44)$. Cette observation s'explique certainement par la faible incidence de la maladie, l'éventualité d'une exposition à l'infection augmentant avec le temps.

La taille du cheptel n'est pas un facteur de risque pour la tuberculose bovine, ce qui diffère des résultats obtenus en Erythrée, en Zambie, en Italie et en Grande-Bretagne (5, 8, 14, 24, 30). Cette différence est probablement liée à l'intensification des systèmes d'élevage dans ces pays, l'augmentation de la taille du cheptel entraînant une forte densité animale dans les bâtiments favorables à la transmission de la maladie (25).

Le risque d'infection est plus faible chez les animaux nés dans le troupeau. Cette observation souligne l'importance de la contamination des cheptels par l'acquisition ou la garde d'animaux. Un jeune âge n'est pas un facteur de risque pour l'infection à M. avium, contrairement aux observations réalisées au Burkina Faso, en Côte d'Ivoire et en Mauritanie (11, 38, 43, 44). Cette différence tient probablement à la méthodologie utilisée dans ces études où toute réaction positive à la tuberculine aviaire avait été attribuée à $M$. avium alors qu'une partie des réactions positives a été classée ici comme douteuse ou atypique.

Le risque d'infection par $M$. avium semble augmenter lorsque la surveillance du cheptel n'est pas assurée par l'éleveur mais confiée aux enfants, ce qui pourrait s'expliquer par une conduite moins attentive à l'origine de contacts étroits avec le réservoir (les volailles).
La prise en charge d'animaux appartenant à d'autres éleveurs semble légèrement protectrice, ce qui pourrait s'expliquer par une période d'exposition au risque plus faible chez les animaux introduits tardivement dans le cheptel.

Enfin, le risque augmente avec le nombre de volailles, ce qui souligne l'importance du réservoir domestique dans l'épidémiologie de l'infection.

Un jeune âge est un facteur de risque pour les réactions atypiques, ce qui rejoint les observations effectuées en Afrique occidentale $(11,38,43,44)$. Cette observation rend peu probable l'hypothèse d'infections par M. farcinogenes ou M. avium subsp. paratuberculosis car elles touchent en priorité les animaux les plus âgés (15, 17). Cette forte proportion de réactions atypiques chez les jeunes pourrait être liée à la transmission de mycobactéries responsables d'affections de la mamelle, telle que $M$. acquae, avant le sevrage. Ces bactéries étant peu pathogènes, les veaux auraient ensuite tendance à se décontaminer. Le risque de réaction atypique semble plus élevé chez les animaux en bon état d'entretien, ce qui pourrait tenir à une meilleure réactivité à une stimulation antigénique. Comme pour la tuberculose aviaire, la surveillance du cheptel par les enfants est un facteur de risque. Cette observation tient probablement au mode de conduite, sans que l'on puisse poser d'hypothèse valide sur les pratiques responsables.

\section{Brucellose}

Le risque d'infection ne semble pas augmenter avec l'âge contrairement à ce qui a été décrit au Sri Lanka (39), au Kenya (18), au Ghana (20) et dans le sud du Soudan (16). Cette augmentation du risque d'infection avec l'âge correspond logiquement à une plus grande probabilité d'exposition au risque chez les animaux âgés. Ce schéma pourrait ne pas être valable dans le cas de l'élevage extensif en zone sahélienne, car la brucellose semble se diffuser lentement au sein des cheptels contaminés et plus rapidement d'un cheptel à l'autre. Ce profil épidémiologique suggère une contamination préférentielle au pâturage, probablement au hasard des rencontres avec des matières virulentes (produits d'avortements) abandonnées sur place. Les femelles les plus âgées, généralement en lactation et retenues à proximité des habitations, pourraient être moins soumises au risque d'infection.

L'ethnie semble être un facteur de risque important. Des observations similaires ont été relevées au Sud Soudan et en Afrique occidentale où les cheptels des éleveurs Foulbé étaient les plus atteints $(13,16)$. Cette observation tient probablement à des pratiques d'élevage spécifiques à l'ethnie et favorables à la transmission de la brucellose.

Le risque d'infection semble plus élevé dans les cheptels de villageois, ce qui pourrait être lié au regroupement des cheptels individuels en troupeau collectif pour la conduite au pâturage.

Le risque associé à la surveillance des animaux par des enfants s'explique probablement par un manque d'expérience avec une conduite moins suivie au pâturage ou un défaut d'élimination des matières virulentes lors d'avortements.

La taille du cheptel n'est pas un facteur de risque contrairement à ce qui a été relevé dans divers travaux $(16,26,29)$. Dans les conditions d'élevage du Tchad oriental, l'augmentation du nombre de bovins n'entraîne pas d'augmentation de la densité animale responsable de la diffusion plus rapide de l'infection au sein des cheptels contaminés.

\section{- CONCLUSION}

La situation épidémiologique de la tuberculose et de la brucellose bovines est caractérisée par un décalage entre une faible préva- 
lence individuelle et une prévalence cheptel plus élevée. Ces maladies se développent donc à bas bruit dans de nombreux cheptels, les conditions d'élevage limitant la propagation de l'infection dans les cheptels contaminés.

Dans le contexte du Tchad oriental, l'impact de ces zoonoses sur la productivité des cheptels et la santé publique est donc certainement faible du fait de la rareté des formes évolutives pouvant contaminer l'homme à travers la consommation de lait cru. Leur existence fait cependant courir un risque aux populations humaines, une augmentation du nombre de cas pouvant intervenir lors d'intensification des conditions d'élevage.

Le profil épidémiologique observé permet une lutte fondée sur l'élimination du réservoir en l'associant au contrôle des animaux introduits dans les cheptels assainis. Cette approche pourrait être retenue en cas de développement de la production laitière périurbaine. Des investigations complémentaires devraient être entreprises dans les élevages transhumants du Tchad oriental.

\section{Remerciements}

Les auteurs remercient les éleveurs ainsi que les personnels du laboratoire de Farcha et du projet Aseto pour leur contribution. Le test de fixation du complément a été réalisé grâce à l'appui du professeur Chantal (service de maladies contagieuses, Ecole nationale vétérinaire de Toulouse).

\section{BIBLIOGRAPHIE}

1. ACHA P.N., SZYFRES B., 1989. Zoonoses et maladies transmissibles communes à l'homme et aux animaux, $2^{\mathrm{e}}$ éd. Paris, France, OIE, 1083 p.

2. BLOCH N., DIALLO I., 1991. Enquête sérologique et allergologique sur les bovins au Niger. Revue Elev. Méd. vét. Pays trop., 44 : 117-122.

3. BONSU O.A., LAING E., AKANMORI B.D., 2000. Prevalence of tuberculosis in cattle in the Dangme-West district of Ghana, public health implications. Acta trop., 76: 9-14.

4. BOTHA C.J., WILLIAMSON C.C., 1989. A serological survey of bovine brucellosis in four districts of Bophuthatswana. J. S. Afr. vet. Assoc., 60: 50.

5. COOK A.J., TUCHILI L.M., BUVE A., FOSTER S.D., GODFREY-FAUSETT P., PANDEY G.S., MCADAM K.P., 1996. Human and bovine tuberculosis in the Monze District of Zambia - A cross-sectional study. Br. vet. J., 152: 3-5.

6. COSIVI O., MESLIN F.X., DABORN C.J., GRANGE J.M., 1995. Epidemiology of Mycobacterium bovis infection in animals and humans, with particular reference to Africa. Revue sci. tech., 14: 733-746.

7. DELAFOSSE A., DOUTOUM A.A., 2001. Epidémiologie de la trypanosomose des dromadaires au Tchad oriental. N'djaména, Tchad ministère de l'Elevage, $244 \mathrm{p}$.

8. DENNY G.O., WILESMITH J.W., 1999. Bovine tuberculosis in Northern Ireland: a case-control study of herd risk factors. Vet. Rec., 144: 305-310.

9. Estimation des effectifs du cheptel au Tchad oriental, 1999. N'djaména, Tchad, ministère de l'Elevage, $52 \mathrm{p}$.

10. FERNEY J., CHANTAL J., 1976. Clinical and epidemiological aspects of bovine brucellosis in tropical Africa. Dev. Biol. Stand., 31: 274-278.

11. GIDEL R., ALBERT J.P., RETIF M., 1969. Enquête sur la tuberculose bovine au moyen de tests tuberculiniques dans diverses régions d'Afrique occidentale (Haute Volta et Côte d'Ivoire). Revue Elev. Méd. vét. Pays trop., $22: 337-355$.

12. GIDEL R., ALBERT J.P., LE MAO G., RETIF M., 1974. La brucellose en Afrique occidentale et son incidence sur la santé publique. Résultats de dix enquêtes épidémiologiques effectuées en Côte d'Ivoire, Haute-Volta et Niger de 1970 à 1973. Revue Elev. Méd. vét. Pays trop., 27 : 403-418.

13. GIDEL R., ALBERT J.P., LE MAO G., RETIF M., 1976. Epidemiology of human and animal brucellosis in Western Africa. The results of six studies in the Ivory Coast, Upper Volta, and Nigeria. Dev. Biol. Stand., 31: 187-200.

14. GOODCHILD A.V., CLIFTON-HADLEY R.S., 2001. Cattle-to-cattle transmission of Mycobacterium bovis. Tuberculosis, 81: 23-41.

15. HAMID M.E., MOHAMED G.E., ABU-SAMRA M.T., EL-SANOUSI S.M., BARRI M.E., 1991. Bovine farcy: a clinico-pathological study of the disease and its aetiological Agent. J. comp. Pathol., 105: 287-301.
16. HELLMANN E., STAAK C., BAUMANN M., 1984. Bovine brucellosis among two different cattle populations in Bahr el Ghazal Province of Southern Sudan. Tropenmed. Parasitol., 35: 123-126.

17. JAKOBSEN M.B., ALBAN L., NIELSEN S.S., 2000. A cross-sectional study of paratuberculosis in 1155 Danish dairy cows. Prev. vet. Med., 46: 15-27.

18. KADOHIRA M., MCDERMOTT J.J., SHOUKRI M.M., KYULE M.N., 1997. Variations in the prevalence of antibody to Brucella infection in cattle by farm, area and district in Kenya. Epidemiol. Infect., 118: 35-41.

19. KAZWALA R.R., KAMBARAGE D.M., DABORN C.J., NYANGE J., JIWA S.F., SHARP J.M., 2001. Risk factors associated with the occurrence of bovine tuberculosis in cattle in the Southern Highlands of Tanzania. Vet. Res. Commun., 2: 609-614.

20. KUBUAFOR D.K., AWUMBILA B., AKANMORI B.D., 2000. Seroprevalence of brucellosis in cattle and humans in the Akwapim-south district of Ghana: public health implications. Acta trop., 76: 45-48.

21. La tuberculose, 1990. Lyon, France, Ecoles nationales vétérinaires françaises, $152 \mathrm{p}$.

22. LE MINOR L., VERON M., 1989. Bactériologie médicale, $2^{\mathrm{e}}$ Ed. Paris, France, Médecine-Sciences, $1107 \mathrm{p}$.

23. LIANG K.Y., ZEGER S.L., 1986. Longitudinal data analysis using generalized linear models. Biometrika, 73: 13-22.

24. MARANGON S., MARTINI M., DALLA POZZA M., NETO F., 1998. A case-control study on bovine tuberculosis in the Veneto region (Italy). Prev. vet. Med., 34: 87-95.

25. MORRIS R.S., PFEIFFER D.U., JACKSON R., 1994. The epidemiology of Mycobacterium bovis infections. Vet. Microbiol., 40: 153-177.

26. NICOLETTI P., 1984. The epidemiology of brucellosis in animals. Dev. Biol. Stand., 56: 623-628.

27. OCHOLI R.A., EZEOKOLI C.D., AKEREJOLA O.O., SAROR D.I., 1996. Use of the enzyme-linked immunosorbent assay for screening cattle for Brucella antibodies in Nigeria. Vet. Q., 18: 22-24.

28. OMER M.K., SKJERVE E., HOLSTAD G., WOLDEHIWET Z., MACMILLAN A.P., 2000. Prevalence of antibodies to Brucella spp. in cattle, sheep, goats, horses and camels in the State of Eritrea; influence of husbandry systems. Epidemiol. Infect., 125: 447-453.

29. OMER M.K., SKJERVE E., WOLDEHIWET Z., HOLSTAD G., 2000. Risk factors for Brucella spp. infection in dairy cattle farms in Asmara, State of Eritrea. Prev. vet. Med., 46: 257-265.

30. OMER M.K., SKJERVE E., WOLDEHIWET Z., HOLSTAD G., 2001. A cross-sectional study of bovine tuberculosis in dairy farms in Asmara, Eritrea. Trop. Anim. Health Prod., 33: 295-303.

31. PERREAU P., 1978. Epidémiologie et importance économique des brucelloses en Afrique tropicale. In : $2^{\mathrm{e}}$ Conférence internationale des institutions de médecine vétérinaire tropicale, GTZ, Berlin, octobre 1976, $503 \mathrm{p}$.

32. PRENTICE R., 1988. Correlated binary regression with covariates specific to each binary observation. Biometrics, 44: 1033-1048.

33. QUIRIN R., RASOLOFO V., ANDRIAMBOLOLONA R., RAMBOASOLO A., RASOLONAVALONA T., RAHARISOLO C., RAKOTOARITAHINA H., CHANTEAU S., BOISIER P., 2001. Validity of intradermal tuberculin testing for the screening of bovine tuberculosis in Madagascar. Onderstepoort J. vet. Res., 68: 231-238.

34. Rapport annuel, 1976. N'djaména, Tchad, Laboratoire de Farcha, 282 p. 35. Rapport annuel, 1977. N'djaména, Tchad, Laboratoire de Farcha, 370 p. 36. Rapport annuel, 1978. N'djaména, Tchad, Laboratoire de Farcha, 354 p. 37. Réflexion prospective sur l'élevage au Tchad, 1998. N'djaména, Tchad, ministère de l'Elevage, $77 \mathrm{p}$.

38. REY J.L., 1976. Enquête tuberculinique humaine et animale dans le Yaga (département du Sahel, Haute Volta). Bobo Dioulasso, Haute Volta, Occge, 9 p.

39. SILVA I., DANGOLLA A., KULACHELVY K., 2000. Seroepidemiology of Brucella abortus infection in bovids in Sri Lanka. Prev. vet. Med., 46: 51-59.

40. Situation de la tuberculose et de la brucellose des bovins en zone périurbaine d'Abéché (préfecture du Ouaddaï, Tchad), 2000. N’djaména, Tchad, Laboratoire de Farcha, $49 \mathrm{p}$.

41. TOMA B., DUFOUR B., SANAA M., BENET J.J., ELLIS P., MOUTOU F., LOUZA A., 1996. Epidémiologie appliquée à la lutte collective contre les maladies transmissibles majeures. Maisons-Alfort, France, Aeema, 551 p. 
42. TURKSON P.K., BOADU D.Q., 1992. Epidemiology of bovine brucellosis in the coastal savanna zone of Ghana. Acta trop., 52: 39-43.

43. VILLON A., BONEL J., 1975. Enquête sur la tuberculose humaine et la tuberculose bovine dans les $1^{\mathrm{e}}$ et $2^{\mathrm{e}}$ régions de la république islamique de Mauritanie du 11 mars au 3 avril 1975. Bobo Dioulasso, Haute Volta, Occge, $12 \mathrm{p}$.

44. VILLON A., 1976. Enquête sur la tuberculose bovine dans le Liptako et I'Oudalan (département du Sahel, Haute Volta). Bobo Dioulasso, Haute Volta, Occge, $19 \mathrm{p}$
45. WEINHAUPL I., SCHOPF K.C., KHASCHABI D., KAPAGA A.M., MSAMI H.M., 2000. Investigations on the prevalence of bovine tuberculosis and brucellosis in dairy cattle in Dar es Salaam region and in zebu cattle in Lugoba area, Tanzania. Trop. Anim. Health Prod., 32: 147-154.

46. WERNERY U., KERANI A.A., VIERTEL P., 1979. Bovine brucellosis in the southern regions of the Somali Democratic Republic. Trop. Anim. Health Prod., 11: 31-5.

Reçu le 22.02.2001, accepté le 10.12.2002

\section{Summary}

Delafosse A., Goutard F., Thébaud E. Epidemiology of Bovine Tuberculosis and Brucellosis on the Periphery of Abeche, Chad

This study was carried out to specify the epidemiology of bovine tuberculosis and brucellosis in the dairy basin of Abeche (district of Ouaddai, Eastern Chad). The populations studied for tuberculosis and brucellosis comprised over oneyear-old animals and over one-year-old females only, respectively. The sample was selected with a two-degree survey (village then breeder). The diagnosis of tuberculosis was established by the single intradermal tuberculin test (SIT), followed by the intradermal comparative tuberculin test (ICTT), and that of brucellosis by a serological analysis [the rose bengal test (RBT), followed by the complement fixation test (CFT)]. A questionnaire was systematically filled out to characterize the type of husbandry, and the case history of selected animals was recorded. These factors were included in a multivariate analysis. A total of 848 animals located in 58 herds were tested by SIT, then 151 of them by ICTT. In the studied populations real prevalences of Mycobacterium bovis and $M$. avium infections were estimated at $0.8 \pm 0.6 \%$ and $2.0 \pm 0.9 \%(\alpha=5 \%)$, respectively. Herd prevalences of $M$. bovis and M. avium infections were estimated at $12.4 \pm 8.5 \%$ and $17.8 \pm 9.8 \%$, respectively. With regard to brucellosis, 634 animals located in 56 herds were tested by RBT, and 35 samples from 21 herds by CFT. Brucellosis real prevalence was estimated at $2.6 \pm 1.2 \%(\alpha=5 \%)$ and herd prevalence at $20.0 \pm 10.5 \%$. The main risk factors identified for bovine tuberculosis were an older age, the origin of the animal (birth outside of the herd) and the presence of animals from other breeders left in the care of the herd-person. The main risk factors identified for avian tuberculosis were the number of birds and the attendant type (breeder's children). The main risk factors identified for brucellosis were the ethnic group (Arab) and the attendant type (breeder's children). Bovine tuberculosis and brucellosis seem to develop slowly within many herds, but the conditions of extensive husbandry limit their spread to the boundaries of the contaminated herd. Given this epidemiologic profile, these diseases might be eradicated on condition that animals undergo a control before being introduced into a disease-free herd. Should dairy production develop in Abeche suburbs, this approach could be adopted.

Key words: Cattle - Mycobacterium bovis - Mycobacterium avium - Tuberculosis - Brucellosis - Epidemiology - Chad.

\section{Resumen}

Delafosse A., Goutard F., Thébaud E. Epidemiología de la tuberculosis y de la brucelosis de los bovinos en la zona periurbana de Abeche (Chad)

El presente estudio tiene como objetivo el de precisar la epidemiología de la tuberculosis y de la brucelosis de los bovinos en el valle lechero de Abeche (distrito de Ouaddaï, Chad oriental). La población estudiada está constituída de animales de más de un año para la tuberculosis y de hembras de más de un año para la brucelosis. La muestra se seleccionó gracias a una encuesta a dos niveles (pueblo y luego criador). El diagnóstico se realizó mediante tuberculina intradérmica (simple y luego comparativa) para la tuberculosis y por análisis serológico [prueba del antígeno tampon (EAT), luego fijación de complemento (FC)] para la brucelosis. Se completo sistematicamente un cuestionario destinado a caracterizar la crianza y se llevó a cabo un recuento conmemorativo sobre los animales seleccionados. Estos factores fueron incluídos en un análisis estadístico con variables múltiples. Se examinaron 848 animales, repartidos en 58 hatos con tuberculina intradérmica simple y 151 mediante tuberculina intradérmica comparativa. Las prevalencias reales de las infecciones por Mycobacterium bovis y $M$. avium en la poblacion estudiada fueron evaluadas respectivamente en $0,8 \pm 0,6 \%$ et $2,0 \pm 0,9 \%(\alpha=5 \%)$. La prevalencia por hato a la infección por $M$. bovis fue evaluada en 12,4 $\pm 8,5 \%$, la de la infección por M. avium en $17,8 \pm 9,8 \%$. Para el diagnóstico de la brucelosis, se examinaron mediante EAT 634 animales repartidos en 56 hatos. Se analizaron 35 muestras provenientes de 21 hatos mediante FC. La prevalencia real de la brucelosis se evaluó a 2,6 $\pm 1,2 \%(\alpha=5 \%)$. La prevalencia del hato se estimó a 20,0 $\pm 10,5 \%$. Los principales factores de riesgo identificados para la tuberculosis bovina fueron edad elevada, origen del animal (nacimiento dentro o fuera del hato) y el mantenimiento de animales pertenecientes a otros criadores. Los principales factores de riesgo identificados para la tuberculosis aviar fueron la cantidad de aves y las características de los hijos del criador. Los principales factores de riesgo identificados para la brucelosis fueron el grupo étnico (Arabe) y las características de los hijos del criador. La tuberculosis y la brucelosis bovinas parecen desarrollarse lentamente en una gran cantidad de hatos, las condiciones de cria extensiva limitan la difusión dentro de los hatos contaminados. Este perfil epidemiológico permite una erradicación de estas zoonosis, a condición de que se le asocie un control de los animales antes de la introducción en los hatos sanos. Este enfoque podrá ser retenido en caso de desarrollo de la producción leche en la periferia de Abeche.

Palabras clave: Ganado bovino - Mycobacterium bovis Mycobacterium avium - Tuberculosis - Brucelosis - Epidemiología - Chad. 